\title{
Iterative learning control of nonlinear non-minimum phase systems and its application to system and model inversion
}

Ola Markusson (KTH), Håkan Hjalmarsson (KTH), Mikael Norrlöf

Division of Automatic Control

Department of Electrical Engineering

Linköpings universitet, SE-581 83 Linköping, Sweden

WWW: http://WwW. control.isy.liu.se

E-mail: mino@isy.liu.se

17th June 2002

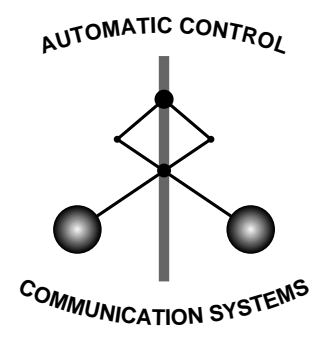

LINKÖPING

Report no.: LiTH-ISY-R-2440

Submitted to 2001 IEEE Conference on Decision and Control, Orlando, Florida, USA.

Technical reports from the Control \& Communication group in Linköping are available at http://www. control.isy.liu.se/publications. 


\begin{abstract}
In this contribution we place ILC in the realm of numerical optimization. This clarifies the role played by the design variables and how they affect e.g. convergence properties. We give a model based interpretation of these design variables and also a sufficient condition for convergence of ILC which is similar in spirit to the sufficient and necessary condition previously derived for linear systems. This condition shows that the desired performance has to be traded against modelling accuracy. Finally, one of the main benefits of ILC when non-minimum phase systems are concerned, the possibility of non-causal control, is given a comprehensive coverage.
\end{abstract}

Keywords: Iterative methods, learning control, nonlinear systems, convergence analysis, non-minimum phase systems 


\section{Iterative learning control of nonlinear non-minimum phase systems and its application to system and model inversion}

\author{
Ola Markusson and Håkan Hjalmarsson \\ Dept. of Signals, Sensors, \& Systems \\ Royal Institute of Technology (KTH) \\ SE-100 44 Stockholm, SWEDEN \\ Tel: +468 7907422 , Fax: +468790 7329 \\ ola.markusson@s3.kth.se hakan.hjalmarsson@s3.kth.se
}

\author{
Mikael Norrlöf \\ Dept. of Electrical Engineering \\ Linköping University \\ SE-581 83 Linköping, SWEDEN \\ Tel: +4613 282704, Fax: +4613 282622 \\ minoهisy.liu.se
}

\begin{abstract}
In this contribution we present a model based method for reference tracking in the Iterative Learning Control (ILC) framework. The method can be applied to nonlinear, possibly non-minimum phase, systems. The idea is to use the inverse of a linearized model in the ILC update. In the non-minimum phase case, the batch property of ILC is explored by means of non-causal filtering. Apart from reference tracking, this method is useful for system and model inversion - a problem that arises in many disciplines where nonlinear systems and models are involved, e.g. maximum likelihood identification and input design for identification for control. The method is illustrated on a numerical example.
\end{abstract}

\section{Introduction}

Iterative learning control (ILC) is typically applied when the reference signal is given over a finite time interval and thereafter repeated. The idea is to use information about the control error from previous iterations to improve the control in the next iteration. An example of such application is a robot arm performing the same task over and over again. Many publications have been written on ILC and (Moore 1993) and (Norrlöf 2000) provide good introductions.

Since most ILC algorithms are based on batch processing they are very well suited for non-causal filtering operations. This property has so far gained very little attention, with a few exceptions. In (Jeong and Choi 2001) a method is presented which uses matrix formulations. However, the method introduces limitations for certain models. In (Sogo et al. 2000) a method is introduced based on time-reversion for maximum phase systems. Another iterative, although cumbersome, method is presented in (Zeng and Hunt 2000).

The method presented here can be used for: 1) Inversion of nonlinear (and linear) non-minimum phase systems and models, 2) Computation of control sequences for nonlinear (and linear) non-minimum phase systems repeatedly following the same reference trajectory. We will consider discrete-time single-input single-output (SISO) systems.

\section{Iterative Learning Control (ILC)}

There are several ILC algorithms and here we use a method depicted in Figure 1. It is important to note that the idea of non-causal filtering presented here can be applied to other forms of ILC as well.

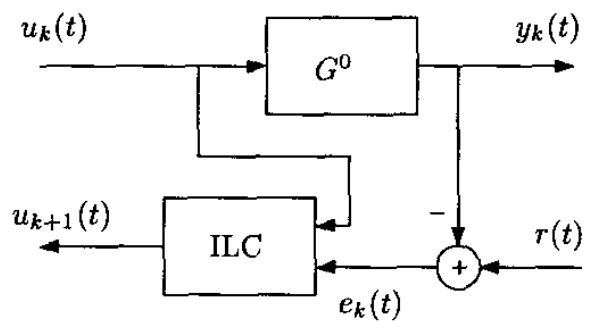

Figure 1: Block diagram of the Iterative learning control (ILC).

The relationships in Figure 1 are given by

$$
\begin{aligned}
& y_{k}(t)=G^{0}\left(u_{k}(t)\right) \\
& e_{k}(t)=r(t)-y_{k}(t)
\end{aligned}
$$

where $t$ denotes time and $k$ is an iteration index. The true nonlinear system is denoted by $G^{0}(\cdot)$, the control signal by $u_{k}(t)$ and $r(t)$ denotes the desired output. The objective of the ILC methods is to iteratively compute the sequence $u_{k+1}(t)$ such that the difference $e_{k}(t)$ between the actual output and the desired output is minimized. The optimal input can be described by

$$
u_{k}(t)=-G^{0}(r(t))^{-1},
$$

where $G^{0}(\cdot)^{-1}$ denotes the inverse of $G^{0}(\cdot)$. As shown in (1) the inverse of the true system is desired but we usually only have access to a simple, typically linear model, $G(q)$ of the system ( $q$ denotes the time-shift operator). The idea of ILC is to compensate for this by iterating on the control signal. Here we consider the updating given by

$$
u_{k+1}(t)=H_{1} u_{k}(t)+H_{2} G^{-1}(q) e_{k}(t)
$$

where $G^{-1}(q)$ denotes the inverse of the linear model $G(q)$. In (2), $H_{1}$ and $H_{2}$ are scalars chosen to achieve stability.

3 ILC for non-minimum phase systems

In (2) the inverse of the model is used to iteratively find the inverse of the system. If the model is nonminimum phase the inverse will be unstable if applied by causal filtering. However, by applying non-causal filtering (exploring the batch nature of ILC) the inverse will be stable. Let us start by considering the linear case. 


\subsection{Inversion of linear systems}

Consider a bounded signal $u(t)$ with $z$-transform $U(z)$ filtered through a stable, albeit not necessarily minimum-phase, linear system $G(z)$ and denote the resulting output $y(t)$ with transform $Y(z)$. Then, $Y(z)=G(z) U(z)$ in the region of convergence which under the given assumptions is a disc which includes the unit disc so that $y(t)$ is bounded. Consequently it holds that $U(z)=G(z)^{-1} Y(z)$. Since both $u$ and $y$ are bounded, $G^{-1}$ is here a stable operator, i.e. its region of convergence includes the unit disc. Hence, when $G(z)$ has zeros outside the unit circle, $G^{-1}(z)$ must be interpreted as a stable but non-causal operator and to recover $u$ from $y$ non-causal filtering has to be performed. This is done as follows: Factorize $G(z)$ as

$$
G(z)=G_{+}(z) G_{-}(z)
$$

where $G_{+}(z)$ includes the minimum phase zeros and $G_{-}(z)$ includes the non-minimum phase zeros. In this case the stable inverse of $G_{+}(z)$ will be causal while the stable inverse of $G_{-}(z)$ is anti-causal and $u$ is recovered by first filtering $y$ through the stable causal filter $G_{+}^{-1}(z)$

$$
w(t)=G_{+}^{-1} y(t)
$$

and then anti-causal filtering of this result

$$
u(t)=G_{-}^{-1} w(t) .
$$

The latter can be performed by filtering the reversed sequence $p(t)=w(N-t)(N$ is the total number of data) through the stable causal filter $v(t)=G_{-}^{-1}\left(z^{-1}\right) p(t)$ and then reversing the result again, i.e. $u(t)=v(N-t)$, see e.g., (Oppenheim and Schafer 1989) for more details.

\subsection{Inversion of nonlinear systems}

If a nonlinear model is available, it may also be used in (2). However, as previously noted, applying the inverse of nonlinear models to (2) can be complicated, especially when the model is non-minimum phase. We therefore suggest that a linear approximation of the system (or model) is used for the inversion in (2). Hence, in the nonlinear case it is the linearized model which is inverted, by the method described in Section 3.1 , and applied to (2).

\section{Numerical example}

In this section we illustrate the proposed method on a nonlinear non-minimum phase system. Consider the nonlinear system $G^{0}(u)$ to be described by

$$
\begin{aligned}
x_{1}(t+1)= & 1.05 x_{1}(t)+1.085 \eta_{1}(t)+1.1450 \eta_{2}(t) \\
& -\frac{x_{1}^{3}(t)}{\left(1+\eta_{1}^{2}(t)\right)}+u(t) \\
\eta_{1}(t+1)= & \eta_{2}(t)-0.05 \eta_{1}(t) \sin \left(x_{1}(t)\right) \\
\eta_{2}(t+1)= & x_{1}(t)+0.6 \eta_{1}(t)+0.7 \eta_{2}(t) \\
y(t)= & x_{1}(t) .
\end{aligned}
$$

which is a third order nonlinear system given in normal form, with a relative degree equal to one (Califano et al. 1998). Linearization of (4), at the origin, gives the linear model

$$
G(z)=\frac{(z+0.5)(z-1.2)}{z^{3}-1.75 z^{2}+1.28 z-0.455}
$$

which has poles inside the unit circle and, as indicated by the zero dynamics, one zero outside the unit circle while the other zero is inside. Inversion of the model was done according to Section 3.1 and the control sequences are computed as in (2) with $H_{1}=1$ and $H_{2}=0.5$. The reference signal $r(t)$ is given by

$$
r(t)=\sin \left(5 \frac{2 \pi t}{N_{s}}\right)+\sin \left(3 \frac{2 \pi t}{N_{s}}\right)+\sin \left(6 \frac{2 \pi t}{N_{s}}\right)
$$

where $N_{s}$ denotes the length of the sinusoidal sequence $\left(N_{s}=510\right)$. Since the system is non-minimum phase the desired output has to be zero in the beginning of the reference sequence in order to allow for non-causal control action. Here the total length of the sequence is $N=540$ samples and the reference is zero for the first 20 and the last 10 samples. The control signal was initiated as $u_{0}(t)=0 \forall t$. The ILC algorithm (2) was updated 20 times and the result is shown in Figure 2.
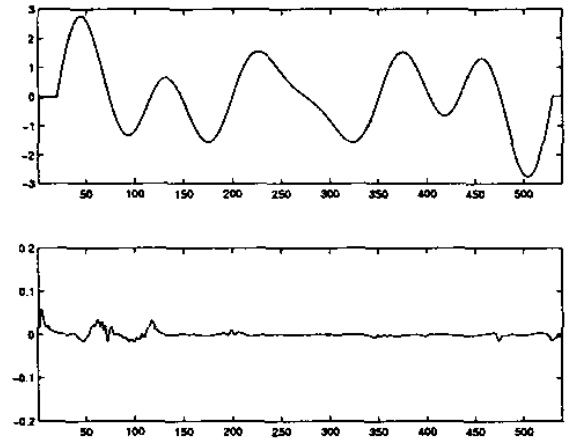

Figure 2: Top: Desired output (solid line) and the system output (dashed line, almost on top of the solid line) . Bottom: Difference between the desired and the actual output $\left(e_{20}(t)\right)$, thus the result after 20 iterations of (2).

\section{References}

Califano, C., S. Monaco and D. Normand-Cyrot (1998). 'On the discrete-time normal form'. IEEE Transactions on Automatic Control 43, 1654-1658.

Jeong, G. and C.H. Choi (2001). Iterative learning control with advanced output data for nonminimum phase systems. In 'Proceedings of the American Control Conference (ACC)'.

Moore, K. (1993). Iterative learning control for Deterministic Systems, Advances in Industrial Control. SpringerVerlag.

Norrlöf, M. (2000). Iterative Learning Control Analysis, Design and Experiments. PhD thesis. Linköping University. Oppenheim, A. and R.W. Schafer (1989). Discrete-Time Signal Processing. Prentice-Hall.

Sogo, T., K. Kinoshita and N. Adachi (2000). Iterative learning control using adjoint systems for nonlinear nonminimum phase systems. In 'Conference on Decision and Control'. IEEE.

Zeng, G. and L.R. Hunt (2000). 'Stable inversion for nonlinear discrete-time systems'. IEEE Transactions on Automatic Control 45(6), 1216-1220. 